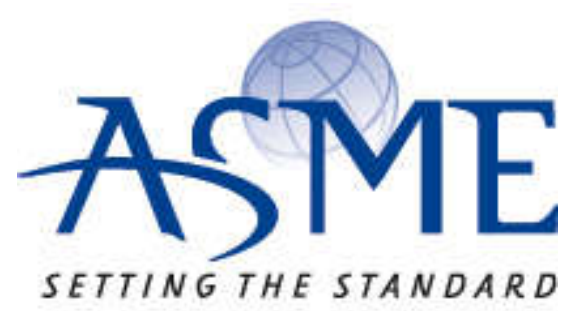

ASME Accepted Manuscript Repository

Institutional Repository Cover Sheet

$\begin{array}{lll}\text { Martin Henke } & \text { Hast }\end{array}$

ASME Paper Title: Introduction of a New Numerical Simulation Tool to

$$
\underline{\text { Analyze Micro Gas Turbine Cycle Dynamics }}
$$

Authors:

Martin Henke, Thomas Monz and Manfred Aigner

ASME Journal Title: Journal of Engineering for Gas Turbines and Power

Volume/Issue _ $139 / 4$

Date of Publication (VOR* Online) Nov 02, 2016

ASME Digital Collection URL: https://gasturbinespower.asmedigitalcollection.asme.org/article.aspx?articleID=2553419

DOI:

*VOR (version of record) 


\title{
Introduction of a New Numerical Simulation Tool to Analyze Micro Gas Turbine Cycle Dynamics
}

\author{
Martin Henke*, Thomas Monz, Manfred Aigner \\ German Aerospace Centre (DLR) \\ Institute of Combustion Technology \\ Pfaffenwaldring 38-40, 70569 Stuttgart, Germany \\ *Email: Martin.Henke@DLR.de
}

\begin{abstract}
Micro gas turbine (MGT) technology is evolving towards a large variety of novel applications, like weak gas electrification, inverted Brayton cycles and fuel cell hybrid cycles; however, many of these systems show very different dynamic behaviors compared to conventional MGTs. In addition, some applications impose more stringent requirements on transient maneuvers, e.g. to limit temperature and pressure gradients in a fuel cell hybrid cycle. Besides providing operational safety, optimizing system dynamics to meet the variable power demand of modern energy markets is also of increasing significance.
\end{abstract}

Numerical cycle simulation programs are crucial tools to analyze these dynamics without endangering the machines, and to meet the challenges of automatic control design. For these tasks, complete cycle simulations of transient maneuvers lasting several minutes need to be calculated. Moreover, sensitivity analysis and optimization of dynamic properties like automatic control systems require many simulation runs. To perform these calculations in an acceptable timeframe, simplified component models based on lumped volume or one-dimensional discretization schemes are necessary. The accuracy of these models can be further improved by parameter identification, as most novel applications are modifications of well-known MGT systems and rely on proven, characterized components.

This paper introduces a modular in-house simulation tool written in Fortran to simulate the dynamic behavior of conventional and novel gas turbine cycles. Thermodynamics, gas composition, heat transfer to the casing and 
surroundings, shaft rotation and control system dynamics as well as mass and heat storage are simulated together to account for their interactions. While the presented models preserve a high level of detail, they also enable calculation speeds up to five times faster than real-time.

The simulation tool is explained in detail, including a description of all component models, coupling of the elements and the ODE-solver. Finally, validation results of the simulator based on measurement data from the DLR Turbec T100 recuperated MGT test rig are presented, including cold start-up and shutdown maneuvers. 


\section{Alphanumeric Variables}

A Cross sectional area $\left[\mathrm{m}^{2}\right]$

C Overall heat capacity $[\mathrm{J} / \mathrm{K}]$

w Mass fraction $[\mathrm{kg} / \mathrm{kg}]$

$c_{f} \quad$ Darcy friction factor [-]

$c_{p} \quad$ Specific isobar heat capacity $[\mathrm{J} / \mathrm{kg} / \mathrm{K}]$

$D \quad$ Hydraulic diameter [m]

$d \quad$ Wall thickness [m]

$h \quad$ Specific enthalpy $[\mathrm{J} / \mathrm{kg}]$

I Moment of inertia $\left[\mathrm{kg} \cdot \mathrm{m}^{2}\right]$

$L \quad$ Component Length [m]

$m \quad$ Mass $[\mathrm{kg}]$

$\dot{m} \quad$ Mass flow $[\mathrm{kg} / \mathrm{s}]$

$N \quad$ shaft speed [1/s]

$\mathrm{Nu} \quad$ Nusselt number [-]

OV Opening value [-]

$P \quad$ Power [W]

$p \quad$ Pressure $[\mathrm{Pa}]$

$R \quad$ Specific gas constant $[\mathrm{J} / \mathrm{kg} / \mathrm{K}]$

$r \quad$ Radius [m]

$\dot{Q} \quad$ Heat flow or work [W]

Re Reynolds number [-]

$S \quad$ Surface area $\left[\mathrm{m}^{2}\right]$

$T \quad$ Temperature $[\mathrm{K}]$

$t \quad$ Time [s]

$U \quad$ Internal energy [J] $u \quad$ Specific internal energy $[\mathrm{J} / \mathrm{kg}]$

$V \quad$ Volume $\left[\mathrm{m}^{3}\right]$

$\dot{V} \quad$ Volumetric flow $\left[\mathrm{m}^{3} / \mathrm{s}\right]$

\section{Greek Variables}

$\alpha \quad$ Overall conduction/convection coefficient $[\mathrm{J} / \mathrm{K}]$

$\delta \quad$ Relative deviation [\%]

$\Delta h_{f}^{0} \quad$ Specific standard enthalpy of formation $[\mathrm{J} / \mathrm{kg}]$

$\Delta p \quad$ Pressure loss $[\mathrm{Pa}]$

$\eta \quad$ Efficiency [\%]

$\lambda \quad$ Thermal conductivity $[\mathrm{W} /(\mathrm{m} \cdot \mathrm{K})]$

$\Pi \quad$ Pressure ratio [-]

$\rho \quad$ Density $\left[\mathrm{kg} / \mathrm{m}^{3}\right]$

$\tau \quad$ Torque $[\mathrm{N} \cdot \mathrm{m}]$

Indices

all Combined conduction/convection

comp Compressor

cond Conduction

conv Convection

el Electrical

exh Exhaust

in At component inlet side

meas Measurement

out At component outlet side

recu Recuperator

rel Relative 


\begin{tabular}{ll} 
sim & Simulation \\
tm & Thermal mass \\
turb & Turbine \\
Abbreviations \\
CC & Combustion Chamber \\
CHP & Combined Heat and Power \\
DLR & German Aerospace Center \\
FC & Free Convection \\
MGT & Micro Gas Turbine \\
P & Plenum \\
PI & Proportional Integral controller \\
PID & Proportional Integral Derivative controller \\
SOFC & Solid Oxide Fuel Cell \\
TIT & Turbine Inlet Temperature \\
TM & Thermal Mass \\
TOT & Turbine Outlet Temperature \\
\hline
\end{tabular}

\section{Introduction}

Primary energy costs are projected to rise again [1] while ever more ambitious $\mathrm{CO}_{2}$-reduction targets are set $[2,3]$. Hence, the strive for more efficient usage of fossil fuels remains a key target. One well-established way to improve fuel efficiency is to produce electrical power on-side and utilize the waste heat. For while overall efficiency of separated heat and power production in the U.S. reaches $51 \%$ [4], decentralized combined heat and power (CHP) applications achieve up to $90 \%$.

Beside their low pollutant emissions, high fuel flexibility and low maintenance costs [5], micro gas turbines (MGT) are very suitable for CHP purposes, as nearly all waste energy is concentrated in the exhaust gas, and is thus easy to utilize. Furthermore, promising MGT cycle innovations are in development that will increase efficiency and open up new fields of application. Inverted Brayton cycle based MGTs [6], for example, can meet power demands as low as $1 \mathrm{~kW}$ el, or hybrid MGT/Solid Oxide Fuel Cell (SOFC) power plants, with the potential to reach electrical efficiency values above $65 \%$ [7,8].

However, developing new cycles like the hybrid power plant is particularly challenging, as the system dynamics change 
significantly while the operation and safety requirements become more demanding, as the fuel cell is vulnerable to fast changes of pressure and temperature. Consequently, numerical simulation tools become essential to study the dynamics of novel cycles efficiently.

Additionally, real-time simulations allow hardware in the loop experiments. Thus, it becomes feasible to develop different aspects of a cycle independent of each other to reduce development time and complexity. The control system hardware is a common example, which can be connected to the simulation tool instead of the actual machine.

Such a simulation tool has to meet various requirements: Thermodynamics, heat transfer to the casing and surroundings, shaft rotation and control system dynamics must be simulated simultaneously to account for their interactions. Mass and heat storage influence the system behavior, and especially for novel cycles, with low-caloric fuels or steam injection, exact track of gas composition is necessary.

While gas dynamics require short simulation time steps, casing temperature changes are slow. Hence, simulating a cold start-up maneuver requires several million time steps. Consequently, simplified models have to be employed to reach appropriate simulation speeds, especially if real-time calculation speed is required. Thus, a common approach is to develop different tools or tool configurations to address different subsets of the listed requirements. One very detailed, to accurately analyze all significant dynamics, and a simplified one, to reach real-time calculation speed [9-12].

This paper introduces a Fortran, in-house simulation code, developed at DLR to meet all requirements listed above while, at the same time, reaching real-time simulation speed. It is based on a consistent physical model, able to simulate all maneuvers including cold start-up and shutdown. At first, the structure and implemented models are introduced. Here, a special emphasis has been placed on accurate Volume discretization and highly conservative energy equations. Sequentially, the modeling of a recuperated Turbec T100 MGT is described. To conclude, validation results are discussed based on simulation results and measurement data from the DLR MGT test rig.

\section{Simulation Tool}

A lumped parameter model approach is used to achieve high simulation speed. Following this approach, it is assumed that all properties are homogeneous within a component, such as temperature of a gas volume or casing part. The recuperator being an exception, as its temperature profile is discretized in flow direction. Complexity and calculation time is further reduced by neglecting the difference between static and total values of thermodynamic quantities. The assumption is reasonable for many MGTs, as flow velocities outside turbocharger components are kept below a Mach number of 0.3 to minimize pressure losses. While gas velocities inside turbocharger components are higher, the implemented model is based 
on turbocharger maps, which account for all flow characteristics.

This section is divided into three parts. At first, the general approach on modularity and module interconnection is explained as well as the integration scheme. Then, the overarching concept of gas flow and storage modules is discussed. Sequentially, all modules are introduced.

\subsection{Modularity and Solver}

To model a MGT, it is divided into its components. Each component is further divided into modules, which are grouped into two categories: Flow modules that only calculate process functions like heat, mass flow or work, and storage modules that are restricted to the calculation of state variables, like temperature, density or rotational speed. The two module types are always connected in an alternating sequence. This approach prevents algebraic constraints and dependencies between modules, which can otherwise only be solved by iterative computation. Thus, it enables higher calculation speed and eases the coupling and implementation of modules.

An example would be the heat flow calculation from the middle of a casing part to ambient air. If one flow module is calculating convective and another one conductive heat flows, a storage module is needed to consider the state variable of surface temperature. Without the storage module, convective and conductive heat must be equal, and the two module equations must be solved iteratively.

While the equations are spread across many different modules, all gradients are stored in a shared interface, which simplifies the development and exchange of solver modules. The implemented solver uses an explicit predictor corrector approach. The second order Adams-Bashforth method is used to predict the state of the next time step. Sequentially, the second order Adams-Moulton approach is used as a corrector with the current and predicted state as inputs. The corrector step is iterated until it converges or hits an iteration limit.

\subsection{General properties of gas flow and storage modules}

The gas flow modules: pipe, combustor, valve, turbo component and recuperator are similar in many aspects. They are interconnected via plena, which are gas storage modules. The flow modules transfer mass, species and energy from one plenum to the next. While mass is transferred through the gas flow module directly, energy and species composition might change in a quasi-stationary way in accordance to the gas flow module's functionality.

Pipe, combustor and recuperator are modelled as flow modules. However, they are exceptions, as they also calculate 
their mass flow rate, which is a state variable:

$$
\frac{\mathrm{d} \dot{m}}{\mathrm{~d} t}=\frac{A}{L} \cdot\left(p_{\text {in }}-p_{\text {out }}-\Delta p\right)
$$

with the cross sectional area $A$, the component length $L$, the pressure of the plena at inlet $p_{\text {in }}$ and outlet $p_{\text {out }}$ and the pressure drop $\Delta p$ of the gas flow module. Sequential calculation is still supported, as pressure and mass flow rate are not directly coupled but via differential equations.

Figure 1 illustrates the interconnection of three pipes (rectangles) with two plena (ovals). The gas volume of the pipe

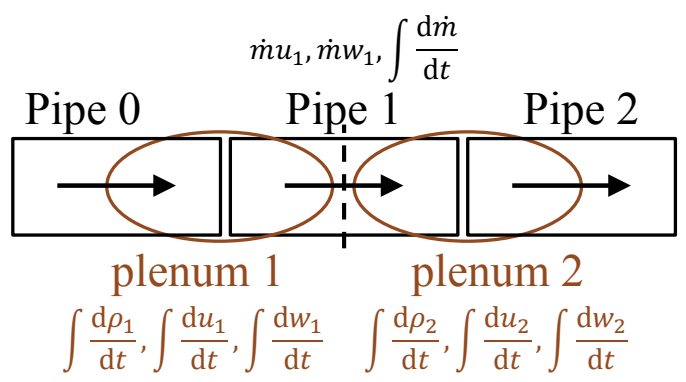

Fig. 1. Illustration of gas phase module interconnection of three adiabatic pipes via two plena (arrow indicates flow direction; variables in nomenclature)

module is equal to the volume of the modelled physical pipe. The volume of a plenum is half the combined volume of all flow modules connected to it. As only plena are calculating energy, species and mass storage, and only flow modules are calculating mass flow rate, there is no need to divide the physical volume among them. The whole volume is assigned to both, and thus is correctly used in the calculation of all states.

As indicated by Fig. 1, the gas phase properties in a gas flow module are calculated by mixing gas from the up- and downstream plena. The mixing ratio is chosen accordingly to the function of the flow module. Combustion heat calculation, for instance, is based on the inlet gas composition and only gas properties of the upstream plena are relevant. In contrast, to calculate heat losses of a pipe, the mean gas temperature must be known, which equals the mixing temperature of both plena.

The gas phase's properties are assumed to be ideal. Fourth-order polynomials of the gas temperature are used for heat capacity [13], conductivity [13] and viscosity [14] calculation along with enthalpy of formation values from [13].

\subsection{Storage Modules}

As discussed, the plenum module is connected to one or several gas flow modules and integrates their energy, species and mass flows. Therefore, a plenum can be used to separate or mix flows. Plena are adiabatic, as heat flows are considered 
in the connected flow modules.

Mass storage for a plenum with $\mathrm{k}$ inflows and $\mathrm{n}$ outflows is calculated by:

$$
\frac{\mathrm{d} \rho}{\mathrm{d} t}=\frac{\sum_{i=1}^{k} \dot{m}_{\text {in }, i}-\sum_{i=1}^{n} \dot{m}_{\text {out }, i}}{V}
$$

with the plenum's Volume $V$. Species gradients can be derived from:

$$
\frac{\mathrm{d} w_{j}}{\mathrm{~d} t}=\frac{\mathrm{d}\left(\frac{m_{j}}{\rho V}\right)}{\mathrm{d} t}=\frac{\mathrm{d} m_{j}}{\mathrm{~d} t} \cdot \frac{1}{\rho V}-\frac{m_{j}}{\rho^{2} V} \cdot \frac{\mathrm{d} \rho}{\mathrm{d} t}=\frac{\sum_{i=1}^{k} \dot{m}_{i n, i}\left(w_{j, i n, i}-w_{j}\right)}{\rho V}
$$

with the variables of species $\mathrm{j}$ : mass $m_{j}=\rho V w_{j}$ and mass fraction $w_{j}$.

The energy equation can be based on temperature. However, deriving the total differential of $T$ is rather complex, considering, for example, the dependency of heat capacity on $T(t)$ and $w(t)$. Hence, it is often implemented in a simplified manner. To remedy this problem and ensure energy conservation, the energy balance is based on the plenum's inner energy. The gradient of inner energy $U$ is given by:

$$
\frac{\mathrm{d} U}{\mathrm{~d} t}=\frac{\mathrm{d} u}{\mathrm{~d} t} \cdot \rho V+\frac{\mathrm{d} m}{\mathrm{~d} t} \cdot u=\sum_{i=1}^{k} \dot{m}_{\text {in }, i} \cdot h_{\text {in }, i}-\sum_{i=1}^{n} \dot{m}_{\text {out }, i} \cdot h_{\text {out }}
$$

from which the gradient of specific inner energy $u$ can be derived:

$$
\frac{\mathrm{d} u}{\mathrm{~d} t}=\frac{\sum_{i=1}^{k} \dot{m}_{\text {in }, i} \cdot h_{\text {in }, i}-\sum_{i=1}^{n} \dot{m}_{\text {out }, i} \cdot h_{\text {out }}-u \cdot\left(\sum_{i=1}^{k} \dot{m}_{\text {in }, i}-\sum_{i=1}^{n} \dot{m}_{\text {out }, i}\right)}{\rho V}
$$

The values can be converted by:

$$
u=h-R T=\Delta h_{f}^{0}+\int_{T=298.15 K}^{T} c_{p}(T) \mathrm{d} T-R T
$$

with the specific standard enthalpy of formation at $298.15 \mathrm{~K} \Delta h_{f}^{0}$ and the specific gas constant $R$.

Thermal mass modules and shaft modules are similar. They integrate their temperature or rotational speed $N$ based on heat flows $\dot{Q}$ respectively torques $\tau$, provided by flow modules. For $\mathrm{n}$ torque values respectively heat flows the gradients are given by:

$$
\begin{aligned}
\frac{\mathrm{d} T}{\mathrm{~d} t} & =\frac{1}{C} \cdot \sum_{i=1}^{n} \dot{Q}_{i} \\
\frac{\mathrm{d} N}{\mathrm{~d} t} & =\frac{1}{2 \pi I} \cdot \sum_{i=1}^{n} \tau_{i}
\end{aligned}
$$


with the overall heat capacity $C$ of the thermal mass and the moment of inertia $I$.

The PID controller module is an implementation of a PID controller with anti-windup [15]. To simulate the behavior of a real PID-microcontroller, the differential equations are not integrated by the common solver. They are implemented as difference equations and solved by the module with a user specified cycle time.

\subsection{Flow Modules}

The pipe module models the gas transport in the MGT with pressure losses and conjugated heat transfer with the thermal mass of the pipes. To determine the dynamic change in mass flow with Eqn. 1 the pressure losses $\Delta p$ are calculated via the Darcy-Weisbach equation:

$$
\Delta p=\frac{c_{f} \cdot L \cdot \rho}{2 \cdot D \cdot A^{2}} \cdot \dot{V}^{2}
$$

With the Darcy friction factor $c_{f}$, the gas density $\rho$, the hydraulic diameter $D$ and the volumetric flow rate $\dot{V}$. Formulas for $c_{f}$ at different pipe flow regimes are implemented as given in [16]. However, to account for complex geometries it is also possible to calculate a fixed $c_{f}$ value based on on-design pressure loss at given flow conditions.

Heat flow from gas phase to thermal mass involves the forced convection at the surface and the conduction to the core of the thermal mass. As all gas phase components and thermal masses are assumed to be tubular shaped the heat flow is given by:

$$
\begin{aligned}
\dot{Q}_{\text {diabatic }} & =\underbrace{\frac{\alpha_{\text {cond }} \cdot \alpha_{\text {conv }}}{\alpha_{\text {cond }}+\alpha_{\text {conv }}}}_{\alpha_{\text {all }}} \cdot\left(T_{\text {gas }}-T_{\text {tm }}\right) \\
\alpha_{\text {cond }} & =\frac{2 \pi \cdot L \cdot \lambda_{t m}}{\ln \left(\frac{D+d}{D}\right)} \\
\alpha_{\text {conv }} & =\frac{N u \cdot \lambda_{\text {gas }} \cdot S}{D}
\end{aligned}
$$

With $\lambda_{t m}$ and $\lambda_{g a s}$ as the thermal conductivity of thermal mass and gas and $T_{t m}$ and $T_{g a s}$ as the corresponding temperatures, $d$ as the thickness of the pipe wall, $S$ for the surface area, $\alpha_{\text {cond }}$ and $\alpha_{\text {cond }}$ for the overall conduction and convection coefficients and $\mathrm{Nu}$ as the Nusselt number. The correlations to calculate $\mathrm{Nu}$ for laminar and turbulent hydrodynamically developed flows in pipes are implemented based on $[14,17]$. For other geometries these correlations can be adjusted by a tuning factor or can be replaced by a user-implemented correlation from Reynolds $(R e)$ and Prandtl to Nusselt number. As the heat flow $\dot{Q}_{\text {diabatic }}$ is directed to the thermal mass, the gas is heated by $\dot{Q}_{\text {heating }}=-\dot{Q}_{\text {diabatic }}$. 
The resulting outlet temperature $T_{\text {outlet }}$ is then derived by solving the implicit equation:

$$
\dot{Q}_{\text {heating }}=\dot{m} \cdot \int_{T_{\text {inlet }}}^{T_{\text {outlet }}} c_{p, \text { out }}(T) \mathrm{d} T
$$

The combustor module is an extension to the pipe module. Fuel and air are not mixed in the combustor module but via the upstream plenum. The mixture enters the combustor module with the composition $w_{i n}$. The enthalpies of formation are given only at $298.15 \mathrm{~K}$. Therefore, the mixture is first cooled down to $298.15 \mathrm{~K}$ :

$$
\dot{Q_{1}}=-\dot{m} \cdot \int_{T_{\text {inlet }}}^{T=298.15 K} c_{p, \text { in }}(T) \mathrm{d} T
$$

The combustion is modeled by the three step mechanism:

$$
\begin{gathered}
\mathrm{C}_{z} \mathrm{H}_{x} \mathrm{O}_{y}+\frac{1}{2}(z-y) \mathrm{O}_{2} \rightarrow z \mathrm{CO}+\frac{x}{2} \mathrm{H}_{2} \\
\mathrm{H}_{2}+\frac{1}{2} \mathrm{O}_{2} \rightarrow \mathrm{H}_{2} \mathrm{O} \\
\mathrm{CO}+\frac{1}{2} \mathrm{O}_{2} \rightarrow \mathrm{CO}_{2}
\end{gathered}
$$

Each step can be restricted with an efficiency value, to account for incomplete combustion. The combustion heat is given by the difference of standard enthalpy of formation between $w_{\text {in }}$ and the exhaust gas composition $w_{\text {out }}$ :

$$
\dot{Q}_{2}=\dot{m} \cdot\left(\Delta h_{f}^{0}\left(w_{\text {in }}\right)-\Delta h_{f}^{0}\left(w_{\text {out }}\right)\right)
$$

The exhaust temperature of a diabatic combustor is calculated analogous to Eqn. (13) with:

$$
\dot{Q}_{1}+\dot{Q}_{2}-\dot{Q}_{\text {diabatic }}=\dot{m} \cdot \int_{T=298.15 K}^{T_{\text {outlet }}} c_{p, \text { out }}(T) \mathrm{d} T
$$

The valve module is equivalent to an adiabatic pipe module, except that the mass flow is derived by a quasi-steady-state assumption. One way to calculate the mass flow is to set an opening value $O V$ between 1 (open) and 0 (closed) and assume the Darcy friction factor to be:

$$
c_{f}=\frac{c_{f 0} \cdot D}{O V^{2} \cdot L}
$$

with the constant $c_{f 0}$ calculated from on-design values. With Eqn. $(1,9)$ and quasi-steady-state assumption $\frac{d \dot{m}}{d t}=0$ follows:

$$
\dot{m}=O V \cdot \sqrt{\frac{2 \cdot \rho \cdot\left(p_{\text {in }}-p_{\text {out }}\right)}{c_{f 0}}}
$$


The other way to control the valve is to input the mass flow directly. A dead-time and PT1 filter delay can be utilized for both demand signals $O V_{\text {setpoint }}$ and $\dot{m}_{\text {setpoint }}$ given by a connected controller.

The turbo component module rely on compressor or turbine maps to calculate the mass flow and temperature change of the gas with a quasi-steady-state approach. The maps are look-up tables used to assign isentropic efficiency $\eta_{i s e n}$ and normalized mass flow rate $\dot{m}_{p}$ according to the pressure ratio $\Pi$ between inlet and outlet plena and the normalized speed of the connected shaft $N_{p}$, with the normalization scheme:

$$
\dot{m}_{p}=\dot{m} \cdot \frac{\sqrt{T_{i n}}}{p_{\text {in }}} ; N_{p}=\frac{N}{\sqrt{T_{\text {in }}}}
$$

For an isentropic pressure change, the outlet temperature $T_{i s e n}$ is calculated by solving the implicit equation:

$$
\int_{T_{\text {in }}}^{T_{\text {isen }}} \frac{c_{p}(T)}{T} \mathrm{~d} T=\int_{p_{\text {in }}}^{p_{\text {out }}} \frac{R}{p} \mathrm{~d} p
$$

With $T_{i s e n}$ as outlet temperature, the isentropic work $\dot{Q}_{i s e n}$ can be derived analogous to $\dot{Q}_{\text {heating }}$ in Eqn. (13). The actual work $\dot{Q}_{\text {actual }}$ is given by multiplying (turbine) or dividing (compressor) $\dot{Q}_{i s e n}$ with $\eta_{i s e n}$. If requested, a heat flow to the casing is subtracted from $\dot{Q}_{a c t u a l}$ to form $Q_{\text {heating. }} T_{\text {outlet }}$ is again calculated by Eqn. (13).

The recuperator module is similar to two pipe modules that are connected to a common thermal mass. However, the calculation of heat flows is more complex. Figure 2 shows a scheme of this calculation. The large rectangle is the recuperator

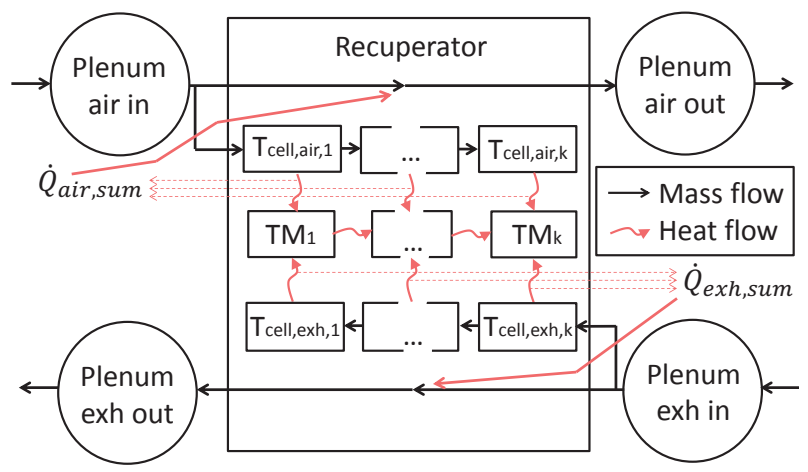

Fig. 2. Recuperator module - heat flow calculation scheme

module, connected to four plena. The two gas flow paths inside the recuperator are led into several thousand thin tubes, based on recuperator core geometry. 
To calculate the temperature profile of the gas inside such a tube, its volume is discretized in flow direction into $\mathrm{k}$ cells with equal length. Each cell calculates a heat flow to the surrounding thermal mass with the same index, representing a part of the heat transfer sheet. Adjacent thermal masses transfer heat also among one another to model conduction along the heat transfer sheets. All heat flows from one gas side to the thermal masses are summed up to calculate the resulting exit temperature of that gas side via Eqn. (13).

The thermal masses integrate their heat flows, developing a dynamic $1 \mathrm{~d}$ temperature profile. However, the calculation of the gas cells are quasi-steady-state, based on the assumption that gas retention time is rather short compared to temperature change rates of the thermal masses.

The heat flow from gas to thermal mass, can be calculated according to Eqn. $(10,12)$ with $\alpha_{c o n d}$ simplified to Eqn. (24) because of the thinness of the heat exchanger sheets and with a user-defined $\mathrm{Nu}$ correlation corresponding to the recuperator core geometry.

$$
\alpha_{\text {cond }}=\frac{2 \cdot \lambda_{t m} \cdot S}{d}
$$

To calculate $\dot{Q}$ via Eqn. (10) the air temperature inside the cell $T_{\text {cell }}$ must be known. $T_{\text {cell }}$ could be assumed to be equal to the known inlet temperature $T_{\text {cell, in }}$. Then the exit temperature $T_{\text {cell,out }}$ could be approximated by:

$$
T_{\text {cell }, \text { out }}=T_{\text {cell,in }}-\frac{\dot{Q}_{\text {diabatic }}}{\dot{m} \cdot c_{p}\left(T_{\text {cell }}\right)}
$$

Subsequently, the oversimplified assumption $T_{\text {cell }}=T_{\text {cell, in }}$ could be improved by:

$$
T_{\text {cell }}=\frac{T_{\text {cell,in }}+T_{\text {cell }, \text { out }}}{2}
$$

However, instead of iteratively solving Eqn. $(10,25,26)$, it is faster and more accurate to analytically solve the system of equations for $T_{\text {cell,out }}$, resulting in:

$$
T_{\text {cell,out }}=T_{\text {cell, in }}-\frac{2 \alpha_{\text {all }}\left(T_{\text {cell,in }}-T_{\text {tm }}\right)}{2 \dot{m} c_{p}+\alpha_{\text {all }}}
$$

The thermal masses can be connected to additional thermal masses, modeling conductive heat flows to the recuperator casing, insulation and free convection to ambient air.

The heat transfer module calculates the conduction of heat between an inner thermal mass to an outer thermal mass. 
Thermal masses are assumed to be tubular shaped (except within the recuperator), which leads to the approximation:

$$
\dot{Q}=\frac{2 \pi \cdot L \cdot \lambda_{\text {tm,inner }} \cdot \lambda_{\text {tm }, \text { outer }} \cdot\left(T_{\text {tm,inner }}-T_{\text {tm }, \text { outer }}\right)}{\ln \left(\frac{r_{\text {tm }, \text { outer }}}{r_{\text {tm,outer }}-d_{\text {tm }, \text { outer }} / 2}\right)+\ln \left(\frac{r_{\text {tm inner }}+d_{\text {tm }, \text { inner }} / 2}{r_{\text {tm,inner }}}\right)}
$$

with radii $r_{\text {tm,inner }}$ and $r_{\text {tm,outer }}$ to the inner and outer thermal mass. Additionally, the module computes the free convection heat flow from a thermal mass to the ambient air by:

$$
\begin{aligned}
\dot{Q} & =\frac{\alpha_{\text {cond }} \cdot \alpha_{\text {conv }}}{\alpha_{\text {cond }}+\alpha_{\text {conv }}} \cdot\left(T_{\text {tm }}-T_{\text {ambient }}\right) \\
\alpha_{\text {cond }} & =\frac{L \cdot 2 \pi \cdot \lambda_{\text {tm }}}{\ln \left(\frac{r_{t m}+d / 2}{r_{t m}}\right)} \\
\alpha_{\text {conv }} & =\frac{N u \cdot \lambda_{\text {ambient }} \cdot S}{L_{\text {chara }}}
\end{aligned}
$$

with the outside surface Area $S$ and the streamed length $L_{\text {chara, }}$, which is the path length of the gas flow along $S$. Nu correlations and calculation of $L_{\text {chara }}$ depend also on pipe mounting position. Equations for horizontal and vertical installation have been implemented and can be found in [17]. $\lambda_{\text {ambient }}$ must be derived based on an average temperature between $T_{\text {ambient }}$ and the surface temperature:

$$
T_{\text {surface }}=T_{\text {tm }}+\left(T_{\text {ambient }}-T_{t m}\right) \cdot \frac{\alpha_{\text {conv }}}{\alpha_{\text {cond }}+\alpha_{\text {conv }}}
$$

Hence, Eqn. (31) and (32) are solved iteratively.

The Generator Module passes a torque value to the shaft, based on breaking power demand $P_{b r e a k}$ and shaft speed $N$. If the generator is air cooled, it passes a fraction of $P_{\text {break }}$, calculated via a linear correlation, to a thermal mass adjacent to the cooling air flow. A part of $P_{\text {break }}$ is lost due to friction:

$$
P_{\text {friction }}=a_{1} \cdot N+a_{2} \cdot N^{2}
$$

with the user defined constants $a_{1}$ and $a_{2}$. Another part from the remaining mechanical power $P_{\text {remain }}$ is lost due to generator and power electrics conversion losses as well as auxiliary systems power demand. These losses are calculated by a user defined third-order polynomial of $P_{\text {remain }}$. 


\section{Validation}

\subsection{Test Rig and Measurements}

The test rig features a Turbec T100 recuperated MGT with detailed instrumentation as described in [18] and [19]. An elongated combustion chamber has been installed to ensure accurate measurement of average turbine inlet temperature [20]. Additionally, ten thermocouples have been installed at each the turbine outlet and recuperator exhaust outlet, to acquire averaged values of the uneven spacial temperature distributions at these locations. The original piping between compressor and recuperator was changed to include an air flow sensor. Consequently, pressure drop and heat capacity of the piping are increased, as shown in Tab. 1.

Two measurement datasets were generated. The utilized air mass flow sensor did not work correctly during start-up and shutdown procedures of the first measurement (dataset 1). Therefore, only the second dataset includes all data from the cold start-up and shutdown sequences. Hence, it was utilized for parameter identification and validation. However, the remaining dynamic load changes in dataset 1 were also used for validation, while this dataset was not used for the identification of system dynamic parameters.

Exhaust gas analysis data of dataset 2 showed incomplete combustion during start-up procedure. Hence, the measured combustion efficiencies were used as simulation boundary conditions to account for the effect. Pressure drop in the combustion chamber was also lower than usual, as shown in Tab. 1, which was probably due to internal leakages in the elongated burner tube. Apart from the start-up procedure, no decrease of combustion efficiency was observed.

\subsection{Turbec T100 MGT Model}

Figure 3 gives an overview of the T100 model. The input of the model is given by five boundary conditions (BC) and combustion efficiencies for the start-up maneuver of dataset 2. Three BCs represent properties of ambient air and high pressure fuel gas. The other two BCs represent the set points of shaft speed and turbine outlet temperature (TOT). For validation proposes, these $\mathrm{BCs}$ are given by measurement data.

The model is similar to a generic recuperated Brayton cycle, except for some T100 specific model extensions. The generator is not only cooled by an oil cycle but also by inlet air [20]. Therefore, its casing is modeled as a thermal mass (TM) connecting generator and inlet cabined (Pipe) via heat flows, as illustrated in the lower left part of Fig. 3. The turbine volute is cooled via preheated air from the recuperator. It is represented via a TM connecting the exhaust gas pipe with the pipe upstream of the combustion chamber (CC) as shown in the middle right part of Fig. 3. Hence, the TM connected to the turbine models not the heat capacity of the volute but only the capacity of the turbine wheel. The physical pipe upstream of 


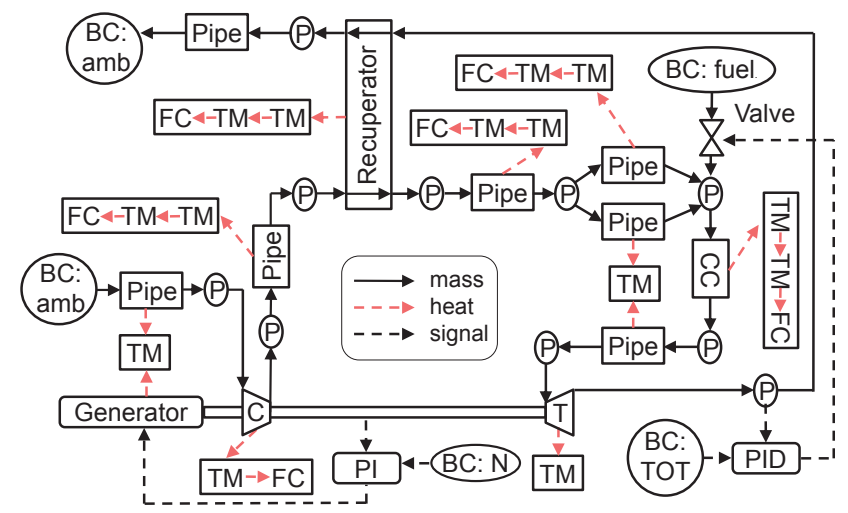

Fig. 3. Scheme of the T100 MGT model $(B C=$ boundary condition; $P=$ plenum; $C C=$ combustion chamber; $T=$ turbine; $C=$ compressor;

$\mathrm{TM}=$ thermal mass; $\mathrm{FC}=$ free convection; $\mathrm{PI} / \mathrm{PID}=$ controller $)$

the CC was divided into two pipe modules to account both for the heat flow to turbine volute and to outer casing.

Each insulated casing is modeled by two thermal masses to account for their difference in heat conductivity and capacity. Thus, the depiction $\mathrm{TM} \rightarrow \mathrm{TM} \rightarrow \mathrm{FC}$ (free convection) is used. The MGT control system consists of two automatic controllers that influence fuel mass flow and generator breaking power to meet a TOT and shaft speed set point. The corresponding controller modules are illustrated in the lower middle and lower right of Fig. 3 as PID and PI.

\subsection{Model Parameter Identification}

Many parameters can be calculated based on knowledge of components geometry and properties, like pipe diameters, wall thicknesses and moments of inertia, or can be found in material data sheets, like conductivity or heat capacity values. However, measurement data is necessary to achieve high simulation accuracy, as complex geometries are simplified and modeled with lumped parameters.

The Darcy friction factors $c_{f}$ of all gas flow components were calculated based upon on-design pressure losses shown in Tab. 1. Convection from gas flows to casing walls and free convection to the atmosphere are calculated based on characteristics of straight round pipes. However, as complex geometries can vary significantly from these simple forms, having different surface areas and flow fields, measurements are required to determine multiplicative tuning constants.

The recuperator is not simplified to a straight round pipe. Here, a Nu correlation was found, based on a formula for rounded cross wavy ducts [21], which was tuned with measured recuperator efficiency data to:

$$
N u=0.0125 \cdot R e+0.32
$$




\begin{tabular}{|c|c|c|c|}
\hline recuperator & $\mathrm{c}$ & $=61.9 \mathrm{~kJ} / \mathrm{K}$ & $\begin{array}{l}\Delta p_{\text {rel,air }}=2.04 \% \\
\Delta p_{\text {rel }, \text { exh }}=2.73 \%\end{array}$ \\
\hline combustor & $\mathrm{c}$ & $=14.5 \mathrm{~kJ} / \mathrm{K}$ & $\Delta p_{r e l}=3.7 \%$ \\
\hline pipes (sum) & $\mathrm{c}$ & $=108.5 \mathrm{~kJ} / \mathrm{K}$ & $\Delta p_{r e l}=3.3 \%$ \\
\hline compressor & $\mathrm{c}$ & $=36.7 \mathrm{~kJ} / \mathrm{K}$ & \\
\hline turbine & $\mathrm{c}$ & $=61.1 \mathrm{~kJ} / \mathrm{K}$ & \\
\hline shaft & I & $=0.0083 \mathrm{~kg} \cdot \mathrm{m}^{2}$ & \\
\hline shaft speed PI & $K_{p}$ & $=1080$ & $K_{i}=4235$ \\
\hline TOT PID & $\begin{array}{l}K_{p} \\
K_{d}\end{array}$ & $\begin{array}{l}=6 \cdot 10^{-6} \\
=1.04 \cdot 10^{-6}\end{array}$ & $K_{i}=8.31 \cdot 10^{-6}$ \\
\hline
\end{tabular}

Table 1. Selected model parameters $(c=$ casing heat capacity without outer insulation)

The manufacturer's turbo component maps cover all steady-state operating points. To also cover start-up and shutdown procedures, map data was extrapolated using the program GasTurb 12 [22]. Sequentially, the maps were shifted and linearly scaled to improve consistency between maps and measurement data.

A PI-controller was implemented to follow the measured shaft speed $N$ and a PID-controller to meet the measured TOT values. All control parameters were determined by utilizing Ziegler-Nichols closed-loop tuning method [23]. The parameters are given in Tab. 1 using the notation for parallel PID structures.

The model of friction and conversion losses in the generator, power electronics and auxiliary components was introduced in the simulation tool section. It was parameterized to meet the measured electrical power output by:

$$
\begin{aligned}
P_{\text {friction }}= & 1.28 \mathrm{Ws} \cdot N \\
P_{\text {conversion }}= & 3473 \mathrm{~W}+0.0812 \cdot P_{\text {remain }}+2.67 \cdot 10^{-7} \frac{1}{W} \cdot P_{\text {remain }}^{2} \\
& -4.48 \cdot 10^{-12} \frac{1}{W^{2}} \cdot P_{\text {remain }}^{3}
\end{aligned}
$$




\subsection{Validation Results}

To describe the quality of simulation results, the relative simulation deviation of a signal $\mathrm{X}$ is defined as:

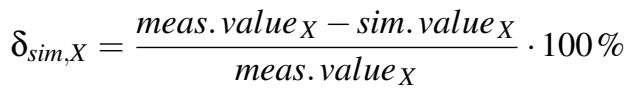

At first, the steady-state validation results are discussed. Figure 4 shows a comparison of simulated (dots) and measured (triangles) air and fuel mass flows, plotted over turbine speed normalized by the full load speed of 70,000 rpm. Air and fuel flow values are labeled to be distinguishable and assigned to the two different ordinates. The average deviation of air mass

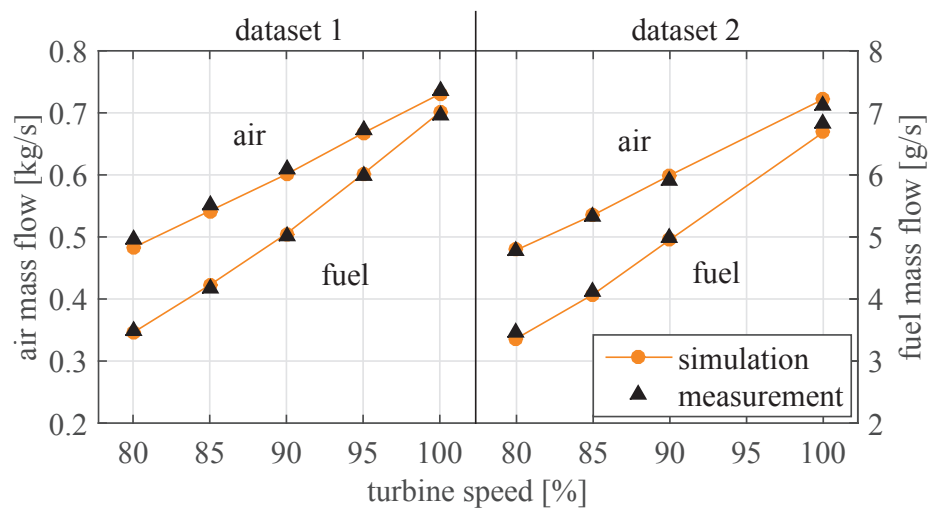

Fig. 4. Steady-state validation results of air and fuel mass flows at various operation points

flow $\delta_{\text {sim,m_air,mean }}$ is $1.2 \%$, with a peak deviation $\delta_{\text {sim,m_air,max }}$ of $2.5 \%$. This reflects the accuracies of the pressure loss model and turbo component maps. Fuel demand depends on many factors such as air mass flow, heat losses and efficiency values of recuperator, compressor, turbine and combustor. The maximum fuel flow deviation is slightly higher as the air flow one with a $\delta_{\text {sim,m_fuel,max }}$ of $3.1 \%$. However, the mean deviation is equally low at $\delta_{\text {sim, } m_{-} \text {fuel,mean }}=1.2 \%$, indicating a good agreement of heat losses and efficiency values to the physical ones.

Additional deviation data are listed in Tab. 2. The deviation of $T_{\text {turb,out }}$ is zero, as $T_{\text {turb,out }}$ is a boundary condition for the control system.

Figure 5 has the same structure as Fig. 4 but shows electrical power output $P_{e l}$ and electrical efficiency $\eta_{e l}$. The correlations given in Eqn. (35) and (36) result in a good agreement of measured and simulated $P_{e l}$ with a $\delta_{\text {sim,Pel,mean }}$ of $1.6 \%$ and $\delta_{s i m, P e l, m a x}$ of $-2.8 \%$. This deviation, combined with the deviation of fuel mass flow, results in $\eta_{e l}$ deviations of $\delta_{\text {sim, }, \text { el,mean }}=1.6 \%$ and $\delta_{\text {sim, }, \text { } e l, \text { max }}=-3.5 \%$.

Measurement data was recorded with a sampling rate of $2 \mathrm{~Hz}$. Hence, the validation of system dynamics is focused on 


\begin{tabular}{lll|lll}
$\delta[\%]$ & mean & $\max$ & $\delta[\%]$ & $\operatorname{mean}$ & $\max$ \\
\hline$T_{\text {comp }, \text { out }}$ & 0.4 & 0.9 & $T_{\text {recu,air,out }}$ & 0.1 & 0.4 \\
$T_{\text {turb,in }}$ & 0.6 & 1.3 & $T_{\text {turb,out }}$ & 0 & 0 \\
$T_{\text {recu, }, \text { exh,out }}$ & 1.5 & 2 & $\eta_{\text {recu }}$ & 0.4 & 0.9 \\
$\Pi_{\text {comp }}$ & 0.4 & 1.0 & $\Pi_{\text {turb }}$ & 0.5 & 1.0
\end{tabular}

Table 2. Additional relative deviation results of steady-state validation $(\Pi=$ pressure ratio)

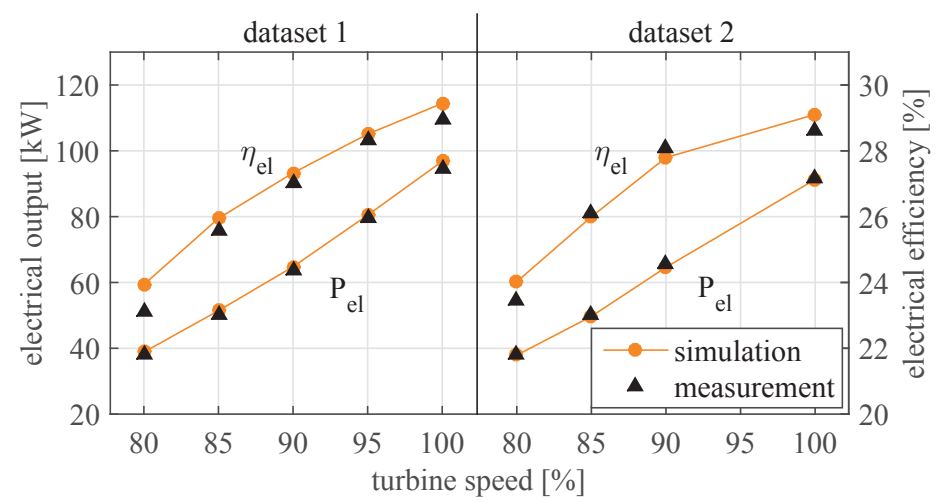

Fig. 5. Steady-state validation results of electrical power output and electrical efficiency at various operation points

effects caused by the MGTs thermal inertias, which dominate the slow dynamics of cold start-up and shutdown maneuvers. All simulations have been done with regular and with 1000 times reduced thermal inertias, to further illustrate their impact on MGT dynamics.

Figure 6 shows a comparison of dataset 1 measurements and simulation results. The upper part of the figure gives an overview of all load changes from $80 \%$ to $100 \%$ of maximum shaft speed in $5 \%$ steps. The plot below shows a magnified view of the upper plot. While a steady temperature deviation of about $2 \%$ is evident, the dynamic response to the load change is very similar. The actual change in shaft speed takes less than $100 \mathrm{~s}$, which is reflected in the simulation results with lower thermal capacities.

Validation data from the cold start-up maneuver is illustrated in Fig. 7. The fuel mass flow of the MGT is plotted in the upper part. At the beginning, ignition is supported by a high mass flow peak, which does not occur in the simulation, as the ignition process is not considered by the lumped volume combustor model. During the fast ramp-up phase, when shaft speed is accelerated from $35 \%$ to $75 \%$, the simulated fuel demand does exhibit an overshoot. As this peak is also visible 

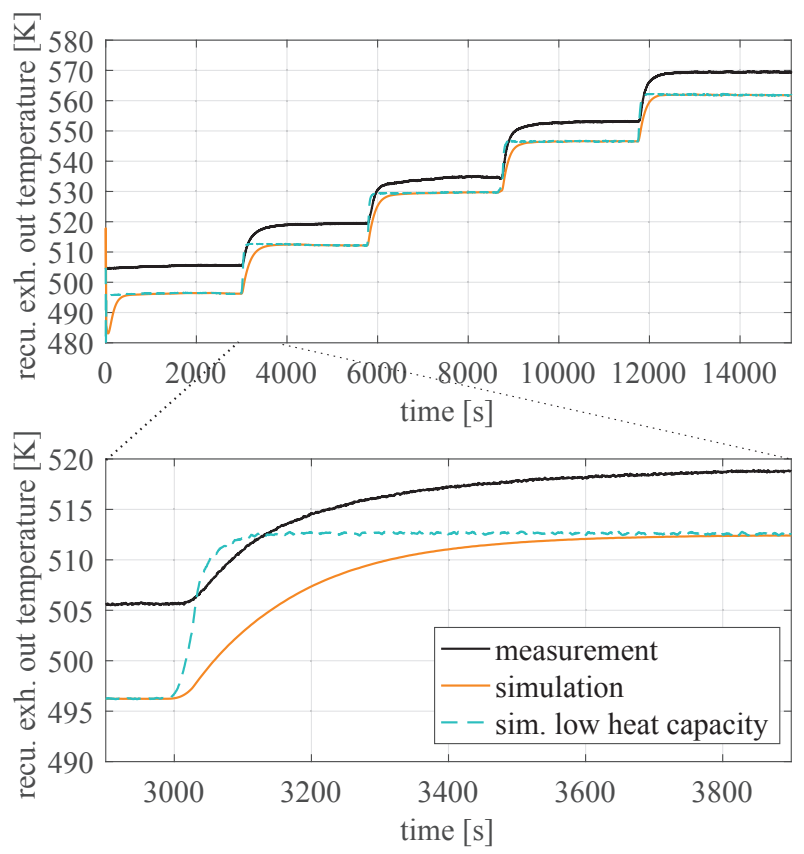

Fig. 6. Recuperator exhaust outlet temperature from measurement dataset 1 and simulation results with full and with low casing heat capacities; shaft speed increased from $80 \%$ to $100 \%$ in $5 \%$ steps (upper part) and magnified detail (lower part)

in the simulation with low thermal inertia, it is presumed to be caused by an overcompensation of the combustor efficiency decrease, described in the measurement section. Apart from this, the shapes of simulated and measured temperature sequence are very similar.

The lower plot in Fig. 7 shows a comparison of the measured and simulated temperature of compressed air at the recuperator outlet. If thermal inertias are neglected, this temperature mainly depends on turbine outlet temperature. Considering thermal inertias, the simulated temperature time sequence is strongly damped and in accordance with the measurement data.

Figure 8 shows the shutdown procedure. While it would be possible to define the maneuver via a decreasing turbine outlet temperature (TOT) ramp, the actual maneuver is initiated by an abrupt fuel cut-off, followed by a decreasing shaft speed trajectory, controlled via generator breaking power. Consequently, the control scheme was adopted for the simulation, using measured fuel mass flow as a set point instead of TOT, to allow TOT deviations.

The upper plot of Fig. 8 shows the gas temperature at recuperator air outlet. After fuel cut-off the temperature decreases slowly, due to the incoming heat flows from the hot recuperator material and other casing parts. While the temperature deviation between measurement and simulation is about $30 \mathrm{~K}$ at the recuperator air outlet and $40 \mathrm{~K}$ at the turbine outlet, the shape of the curves are in good agreement.

Due to the thermal inertias, the generator does still extract work from the MGT for approximately $90 \mathrm{~s}$ after the fuel 

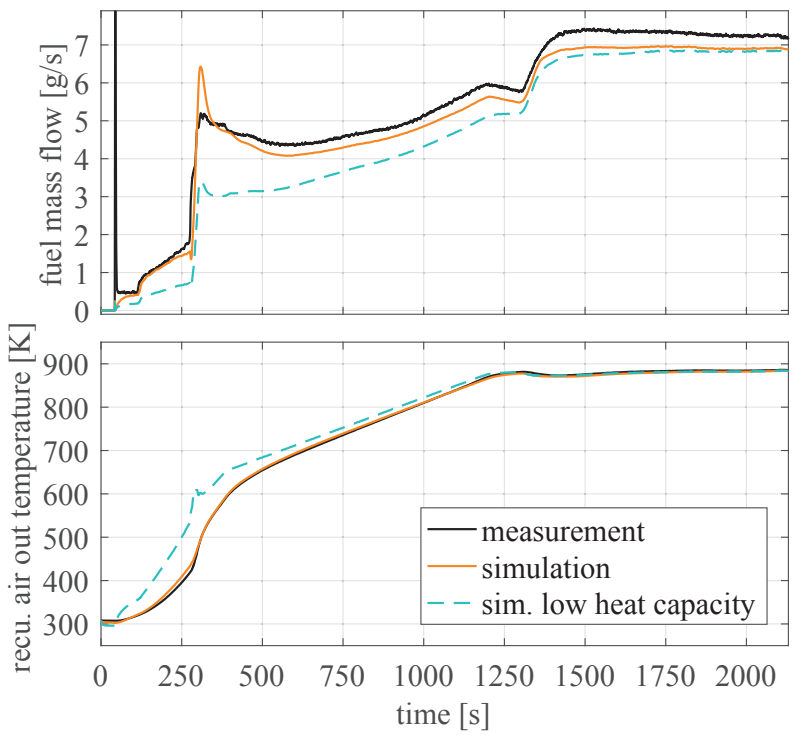

Fig. 7. Measurement data of cold start procedure and simulation results with full and with low casing heat capacities; recuperator air outlet temperature (upper part) and fuel mass flow (lower part)

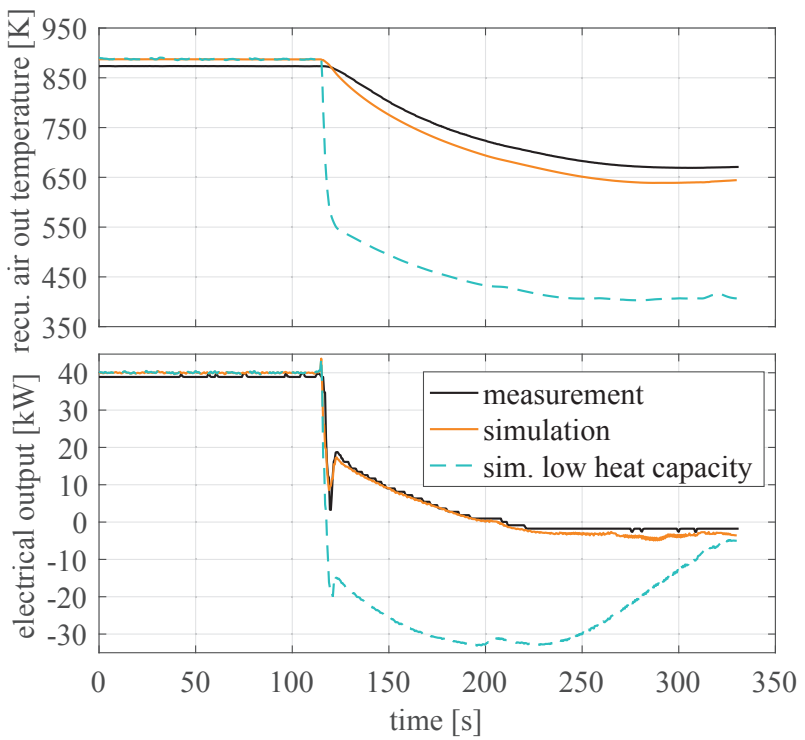

Fig. 8. Measurement data of shutdown procedure and simulation results with full and with low casing heat capacities; recuperator air outlet temperature (upper part) and electrical output power (lower part)

cut-off, as illustrated in the lower plot of Fig. 8. The phenomenon is very accurately reproduced by the simulation data. 


\subsection{Time Step and Calculation Speed}

A time step of $0.5 \mathrm{~ms}$ was used for all simulations. The limiting factor is given by the dynamics of gas flow modules, with smallest gas volumes of about 10 liters. The simulations were done using an Intel Core i7-4790 CPU achieving a ratio of simulated time to calculation time of about 6.

If only slower dynamics, like thermal inertias, are of interest, the volumes can be artificially enlarged to allow longer time steps. As a trial, the simulations were run again with a minimal volume limit of 100 liter. While this method introduces an error on gas transport delay and storage capacity, the impact on slower dynamics is negligible. The time step of these simulations was set to $5 \mathrm{~ms}$, enabling a ratio of simulated time to calculation time of about 50 .

\section{Conclusion}

A simulation tool has been presented, developed to analyze micro gas turbine cycle dynamics. The discretization scheme, approach on modularity, module interconnection and solver have been discussed in detail. Sequentially, the implemented model equations of all components are presented, modeling thermodynamics, conjugated heat transfer between gas and casing as well as shaft and control system dynamics.

A model of the Turbec T100 recuperated MGT was implemented and discussed to validate the simulator with measurement data from the DLR T100 test rig. Validation results of steady-state load points show a high accordance to measurement data, with no mean deviations above $2 \%$. The dynamic behavior was successfully validated with measurement data including cold start-up, load variations and shutdown procedure. Additional, simulation results with lower casing heat capacities were presented, demonstrating their impact on system dynamics.

The implemented T100 MGT model integrates 342 states with a temporal resolution of $0.5 \mathrm{~ms}$. Calculation time was over five times shorter than the simulated time interval. Hence, the tool achieves both development targets: simultaneous simulation of all significant MGT dynamics with high model accuracy while, at the same time, reaching real-time simulation speed.

\section{References}

[1] U.S. Energy Information Administration, 2015. “Annual energy outlook 2015 with projections to 2040”. In DOE/EIA0383(2015).

[2] UNFCCC (United Nations Framework Convention on Climate Change). "Adoption of the paris agreement, fccc/cp/2015/1.9/rev.1". 
[3] Birol, F., 2015. "World energy outlook 2015”. International Energy Agency.

[4] Darrow, K., 2015. "Catalog of chp technologies". U.S. Environmental Protection Agency.

[5] Pilavachi, P., 2002. "Mini-and micro-gas turbines for combined heat and power". Applied Thermal Engineering, 22(18), pp. 2003-2014.

[6] Henke, M., Monz, T., and Aigner, M. "Inverted brayton cycle with exhaust gas recirculation - a numerical investigation”. J Eng Gas Turb Power, 135(9).

[7] Hohloch, M., Huber, A., and Aigner, M. "Experimental investigation of a sofc/mgt hybrid power plant test rig - impact and characterization of a fuel cell emulator". In Proceedings of ASME Turbo Expo 2016, Seoul, South Korea.

[8] McLarty, D., Brouwer, J., and Samuelsen, S., 2013. "Hybrid fuel cell gas turbine system design and optimization". Journal of Fuel Cell Science and Technology, 10(4), p. 041005.

[9] Traverso, A. "Transeo code for the dynamic performance simulation of micro gas turbine cycles". In Proceedings of ASME Turbo Expo 2005, Reno, Nevada, USA.

[10] Damo, U., Ferrari, M., Turan, A., and Massardo, A., 2015. "Test rig for hybrid system emulation: New real-time transient model validated in a wide operative range". Fuel Cells, 15(1), pp. 7-14.

[11] Wächter, C., Lunderstädt, R., and Joos, F., 2006. "Dynamic model of a pressurized sofc/gas turbine hybrid power plant for the development of control concepts". Journal of Fuel Cell Science and Technology, 3(3), pp. 271-279.

[12] Kroll, F., Nielsen, A., and Staudacher, S. “Transient performance and control system design of solid oxide fuel cell/gas turbine hybrids”. In Proceedings of ASME Turbo Expo 2008, Berlin, Germany.

[13] Goos, E., Burcat, A., and Ruscic, B., 2010. "Extended third millennium ideal gas and condensed phase thermochemical database for combustion with updates from active thermochemical tables". URL http://burcat.technion.ac.il/dir/BURCAT.THR.

[14] VDI Gesellschaft, 2006. VDI-Wärmeatlas, 10 ed. Springer Berlin Heidelberg.

[15] Ang, K. H., Chong, G., and Li, Y., 2005. "Pid control system analysis, design, and technology". Control Systems Technology, IEEE Transactions on, 13(4), pp. 559-576.

[16] Bohl, W., 1991. Technische Strömungslehre, 9 ed. Vogel.

[17] Cerbe, G., 2008. Technische Thermodynamik, 15 ed. Hanser.

[18] Hohloch, M., Widenhorn, A., Lebküchner, D., Panne, T., and Aigner, M., 2008. "Micro gas turbine test rig for hybrid power plant application”. In Proceedings of ASME Turbo Expo 2008, Berlin, Germany.

[19] Hohloch, M., Zanger, J., Widenhorn, A., and Aigner, M., 2010. "Experimental characterization of a micro gas turbine 
test rig”. In Proceedings of ASME Turbo Expo 2010, Glasgow, UK.

[20] Henke, M., Klempp, N., Hohloch, M., Monz, T., and Aigner, M. "Validation of a t100 micro gas turbine steady-state simulation tool”. In Proceedings of ASME Turbo Expo 2015, Montréal, Canada.

[21] Utriainen, E., and Sundén, B., 2002. "A numerical investigation of primary surface rounded cross wavy ducts". Heat and Mass transfer, 38(7-8), pp. 537-542.

[22] Kurzke, J. “Gasturb the gas turbine performance simulation program”. URL http://www.gasturb.de.

[23] Ziegler, J. G., and Nichols, N. B., 1942. “Optimum settings for automatic controllers”. trans. ASME, 64(11). 